\title{
Surgical Treatment of Neurofibromatosis-1 with Anterior- Posterior Fusion and Concave Strut Graft for Dystrophic Kyphoscoliosis with Dislocation: A Case Report with 10-Year Follow Up
}

\author{
Jane Lee, BCom, LLB, Noriaki Kawakami, MD, DMSc*, Ryoji Tauchi, MD, DMSc, Kazuki Kawakami, BKin \\ and Tetsuya Ohara, MD
}

Department of Orthopedics and Spine Surgery, Meijo Hospital, Nagoya, Japan

\begin{abstract}
Although dystrophic Neurofibromatosis type-1 (NF-1) is associated with severe deformity, NF-1 with vertebral dislocation only is extremely rare. We present outcomes of a 10-year follow-up of a combined anterior-posterior fusion for the correction of severe dystrophic kyphoscoliosis with a T3/T4 dislocation in a pediatric patient with neurofibromatosis type 1. Two-staged combined posterior-anterior fusion that was performed twice with an interval of four years adequately controlled spinal curvatures over a 10-year period in NF-1 dystrophic kyphoscoliosis. The long-term maintenance of correction despite dystrophic progression is largely attributed to the mechanical support provided by the anterior strut fusion of the concave side of the curvature.
\end{abstract}

Keywords

Neurofibromatosis-1, Scoliosis, Dystrophic kyphoscoliosis, Posterior-anterior fusion, Proximal junctional kyphosis

\section{Introduction}

Neurofibromatosis is a multisystem disorder affecting 1:3000-4000 people encompassing all ethnic groups worldwide [1-3]. Neurofibromatosis type 1 (NF-1), also known as von Recklinghausen neurofibromatosis, is the most common clinical subtype, and although there is significant variability in phenotypic expression, patients frequently present with orthopedic, dermal, and ophthalmological symptoms. Dystrophic type NF-1 is commonly associated with severely progressive scoliosis, kyphosis predominantly in the thoracic spine, and kyphoscoliosis [2]. To the best of our knowledge, only four studies have reported the spontaneous dislocation associated with dystrophic NF-1 with a maximum follow-up of 4 years [4-7]. Although the pathogenesis of the dislocation remains unclear, it might be due to bony erosion secondary to dystrophy or severe curvature deformity causing abnormal distribution of forces. We present a case of NF-1 with a severe dystrophic erosion of the vertebral column causing thoracic dislocation with kyphoscoliosis. The patient was treated with staged combined anterior-posterior surgery with a subsequent 10-year follow-up.

\section{Case Report}

A 10-year-old male patient first presented to the referring institution with the onset of thoracic back pain and de- velopment of a subjective spinal deformity. The patient was observed until the age of 12 years when referral to the treating institution was necessary due to the worsening of presenting symptoms. At hospital presentation, he reported no developmental delays, sensory deficits, or weakness; regular school attendance; and sports participation. He had no immediate family history of NF-1 and no other significant past medical history except for strabismus.

On physical examination, the patient was $133 \mathrm{~cm}$ in height and weighed $32 \mathrm{~kg}$ with a BMI of 18.1. Multiple dermal hyperpigmentation (café-au-lait spots) was present, and although kyphoscoliosis was evident, the patient's external appearance

*Corresponding author: Noriaki Kawakami, MD, DMSc, Director, Department of Orthopedics and Spine Surgery, Meijo Hospital, 1-3-1 Sannomaru, Naka-ku, Nagoya-shi, Aichi-ken, Japan 460-0001, Tel: +81-52-201-5311, Fax: +815-220-105-318

Accepted: June 11, 2019

Published online: June 13, 2019

Citation: Lee J, Kawakami N, Tauchi R, et al. (2019) Surgical Treatment of Neurofibromatosis-1 with Anterior-Posterior Fusion and Concave Strut Graft for Dystrophic Kyphoscoliosis with Dislocation: A Case Report with 10-Year Follow Up. J Orthop Surg Tech 2(1):74-79 
Citation: Lee J, Kawakami N, Tauchi R, et al. (2019) Surgical Treatment of Neurofibromatosis-1 with Anterior-Posterior Fusion and Concave Strut Graft for Dystrophic Kyphoscoliosis with Dislocation: A Case Report with 10-Year Follow Up. J Orthop Surg Tech 2(1):74-79

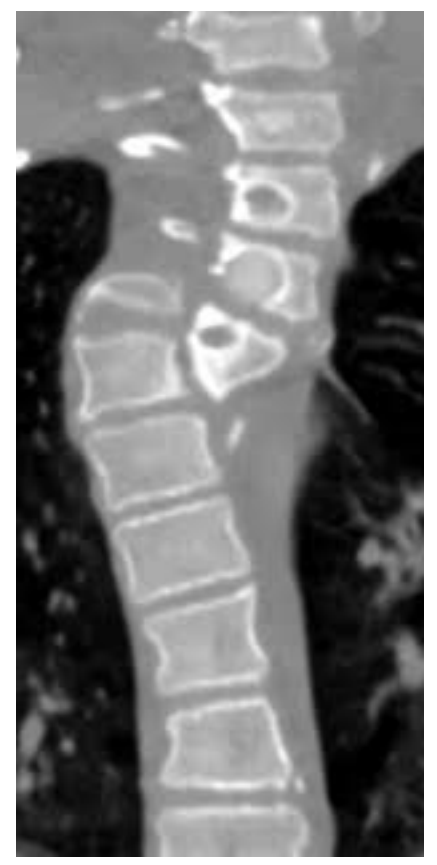

Figure 1: Preoperative CT of T4 dislocation right, superiorly, and dorsally $\mathrm{CT}$, computed tomography. did not accurately reflect its severity. He exhibited a tandem gait, and pulmonary function testing showed a restrictive pattern without dyspnea. Remarkably, no neurological deficits, including hyperreflexia, the loss of power, or sensory disturbance, were noted, and there was no tenderness besides the upper thoracic back pain.

Radiography and MRI revealed severe dystrophic changes including rib penciling, scalloping, neurofibroma, and wedging of the cervicothoracic vertebrae with dural ectasia (Figure 1, Figure 2, and Figure 3). Most notably, he had dislocation at T3/T4 with the dorsal, right, and superior displacement of T4 causing bayonet apposition with T2/T3 (Figure 1). In addition, $64^{\circ}$ of scoliosis from T4-T7 and $56^{\circ}$ of thoracic kyphosis were present (Figure 2). No spinal cord compression was detected on myelogram, CT myelogram, or MRI images except shifting towards the concave side of the apex (Figure 3). Soon after making the strategic decision to perform a two-staged posterior-anterior surgery, halo-gravity traction was applied to correct the kyphosis following curve flexibility assessment. Subsequently, C7-L1 posterior correction and fusion with osteotomy was performed. Severe dystrophic changes at the level of dislocation (T3/T4) and substantial laminal thinning from T2-T9 were noted intraoperatively. Pedicle screws could be
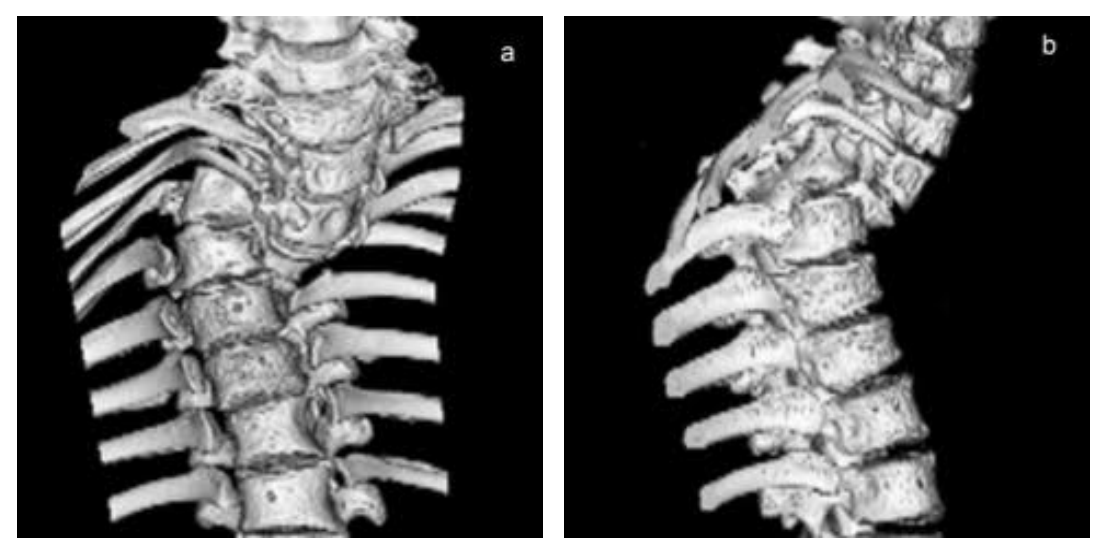

Figure 2: Preoperative 3D CT. $64^{\circ}$ scoliosis (a) and $56^{\circ}$ thoracic kyphosis (b). Note the dystrophic changes with vertebral rotation in the upper thoracic vertebral column.

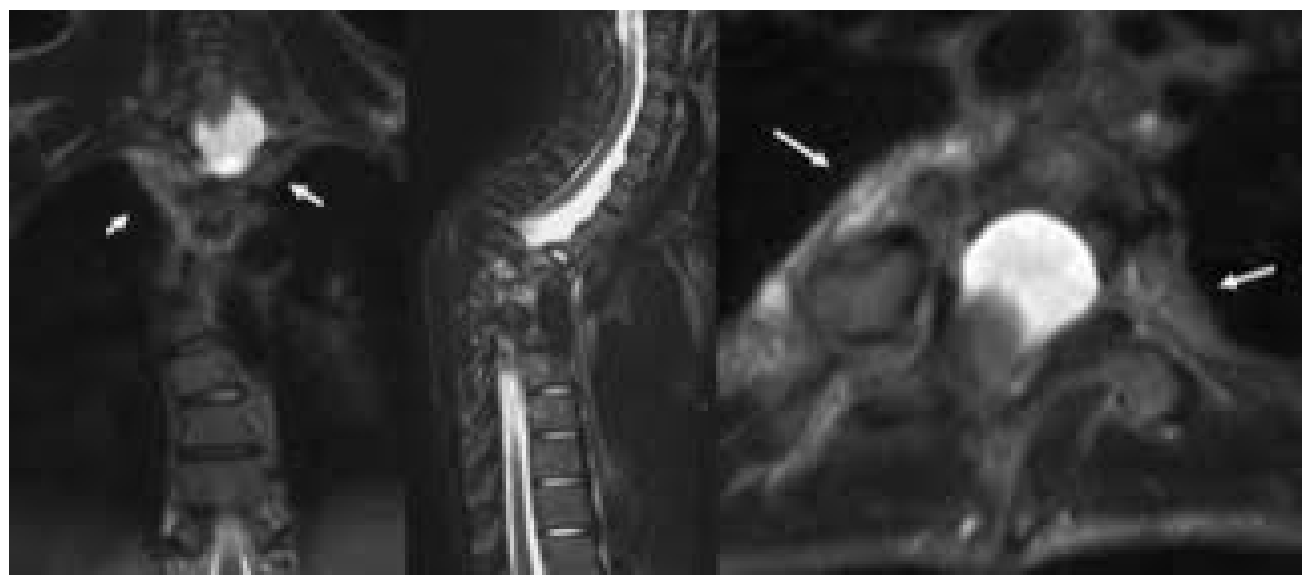

Figure 3: Preoperative MRI T2-wighted images. Note the dystrophic changes with dural ectasia and neurofibroma (arrow) at the paravertebral concave and convex sides. 
Citation: Lee J, Kawakami N, Tauchi R, et al. (2019) Surgical Treatment of Neurofibromatosis-1 with Anterior-Posterior Fusion and Concave Strut Graft for Dystrophic Kyphoscoliosis with Dislocation: A Case Report with 10-Year Follow Up. J Orthop Surg Tech 2(1):74-79
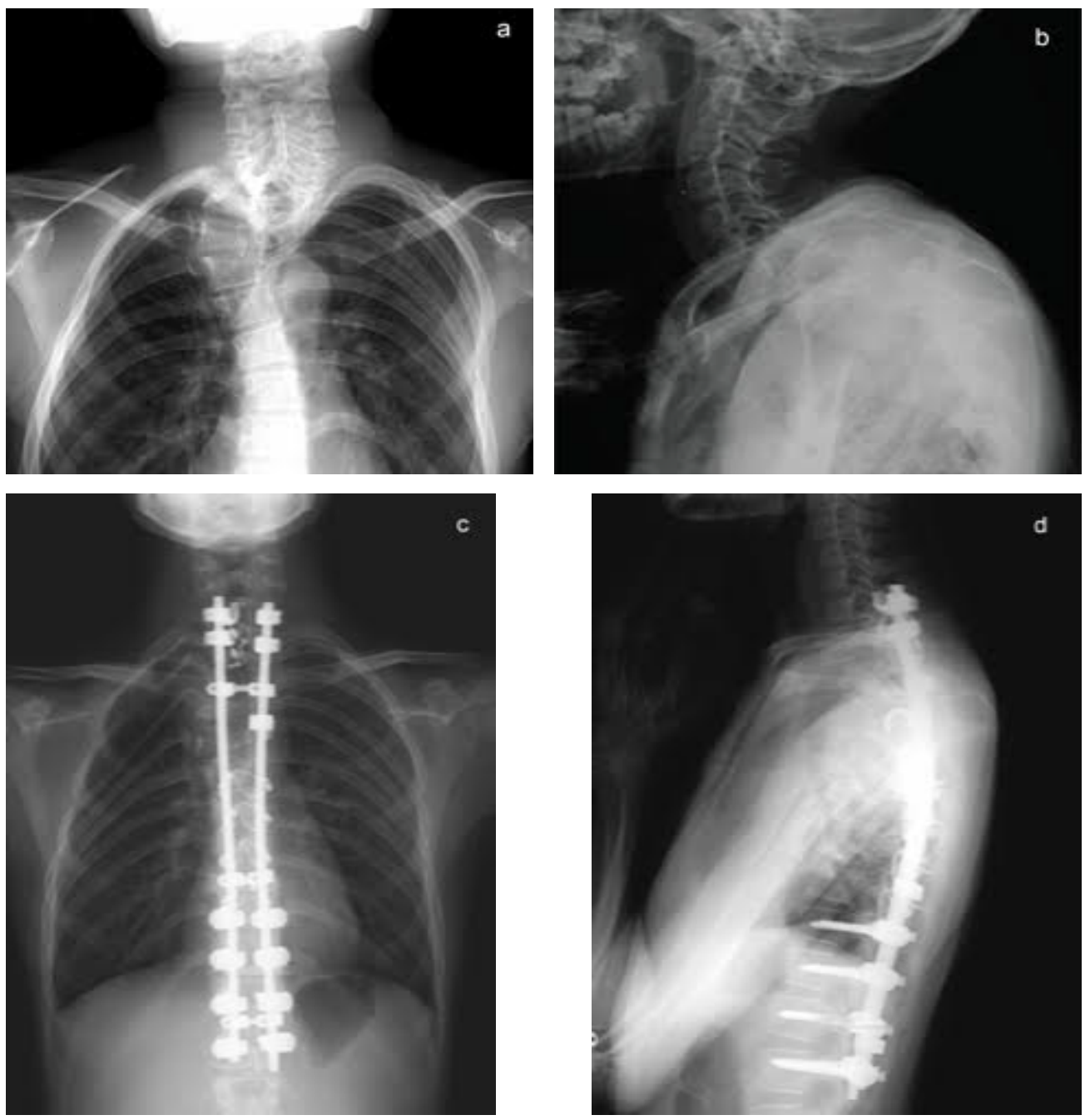

Figure 4: Plain radiographs following C7-T1 posterior osteotomy with instrumentation and T2-T6 anterior fusion with a rib graft. Preoperative coronal (a) and sagittal (b) views show scoliosis of $64^{\circ}$ and thoracic kyphosis of $56^{\circ}$. Postoperative coronal (c) and sagittal (c) views show correction of scoliosis to $24^{\circ}$ and kyphosis to $47^{\circ}$.

inserted only at the T10-L1 segments, and hooks were placed on $\mathrm{C} 7$ and $\mathrm{T} 1$ facet joints with sublaminar wiring to prevent displacement. Osteotomy was performed by resecting the posterior column at T3 and T4 including the pedicles at T4 and the right pedicle at T3. Osteotomy of the anterior column was also performed by removing the $4^{\text {th }}$ rib head and T4 vertebral bodies on the concave (left) side to obtain mobility between T3 and T5. However, the dislocation reduction effort was abandoned due to a dural tear and a drop in electric motor evoked potential (MEP) during the procedure. After confirming MEP recovery, a titanium-alloy rod was placed bilaterally from T1-L1, followed by gradual distraction to correct kyphosis and scoliosis. Mixed morselized allograft and autograft were performed after placing two rods with MEP wave recognition. Rib strut grafting for anterior fusion occurred approximately 3 months following the posterior surgery as the patient experienced mental depression and malnutrition because he refused to eat. During anterior surgery, T2-T6 vertebral bodies were accessed via left thoracotomy, and a plexiform neurofibroma was detected on the dystrophic concave side. Careful partial resection of the neurofibroma was performed to expose the vertebral column, and discectomy was performed from T2-3 to T4-5 including cranial and caudal vertebral endplate removal of $\mathrm{T} 2$ and T6, respectively. On the concave side of the curvature, a rib strut graft was placed from T2-T6 taking care with its orientation in order to maximize structural support and promote osseous union. Postoperative imaging showed significant scoliosis correction to $24^{\circ}$ and kyphosis to $47^{\circ}$ (Figure 4). No neurological deficits were detected during the postoperative period.

However, a gradual loss of cervical lordosis was observed on follow-up imaging in spite of no neck or back complaints from the patient. Four years after anterior-posterior fusion, radiographs showed proximal junctional kyphosis (PJK) of $18^{\circ}$, and MRI displayed progressive dural ectasia causing severe scalloping of the cervicothoracic segments (Figure 5). Accordingly, 2-staged posterior cervical fusion and C3T1 anterior fibula strut graft were performed in which cervical lordosis of $36^{\circ}$ was successfully regained (Figure 6). Furthermore, the cervical-to-thoracic transition zone was augmented by a beta-TCP graft after a second surgery at 2 years to counter the progressive dystrophic change 


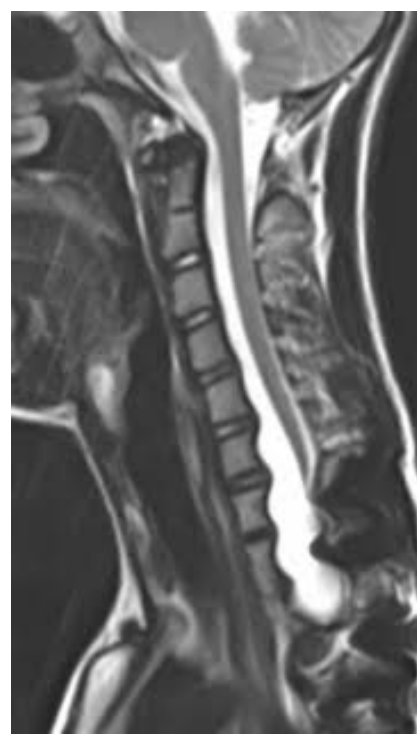

Figure 5: Sagittal T2 MRI shows cervical dural ectasia with posterior vertebral scalloping MRI, magnetic resonance imaging.

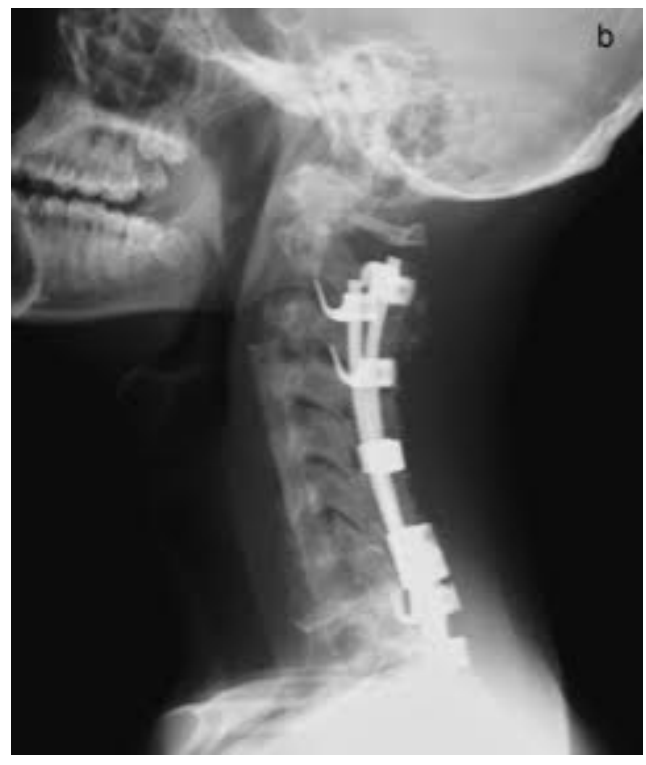

Figure 6: Lateral radiographs (a) pre and (b) post posterior corrective fusion with anterior fibula strut graft from C3-T1 for PJK (18 $8^{\circ}$ kyphosis to $36^{\circ}$ lordosis).

manifesting cervical bone mass thinning. There was no decline in activities of daily living, and the patient was discharged from the second and third surgeries without complications. We used a polymer CTLSO brace to reduce flexion and rotation of the spine for 6 months.

At 10-year follow-up, the patient denied any pain and remained neurologically intact. Scoliosis of $31^{\circ}$, thoracic kyphosis of $49^{\circ}$, and cervical lordosis of $36^{\circ}$ was measured on plain X-ray, showing an $8^{\circ}$ progression of scoliosis, a $2^{\circ}$ loss of thoracic kyphosis correction, and maintenance of cervical lordosis (Figure 7). Dystrophic change progression was not clearly detected; therefore, we will continue to monitor the patient.

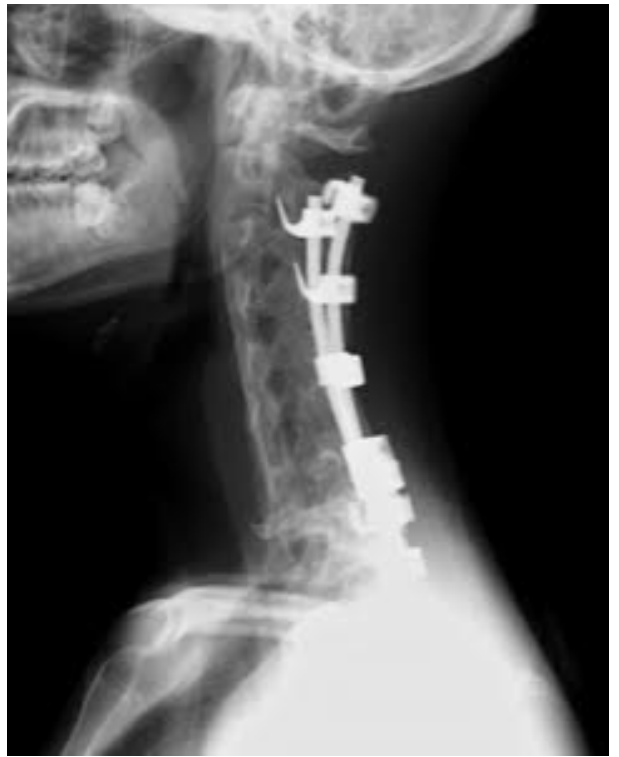

Figure 7: Lateral plain radiograph of cervical spine at the postoperative 10 years. Note solid fusion of fibula grafted in the cervical spine and well-maintained cervical lordosis.

\section{Discussion}

Although dystrophic NF-1 is associated with severe deformity, NF-1 with vertebral dislocation only is extremely rare. Counterintuitively, the lack of neurological deficits is likely a sign of severe dystrophy as dural expansion has caused sufficient vertebral erosion and canal widening to prevent cord compression despite the dislocation $[5,8]$. Currently, surgical stabilization is critical as progression may cause eventual destabilization with neurological compromise.

Our patient presented at age 12 years with kyphoscoliosis of $56^{\circ}$ and $64^{\circ}$, respectively, yet the magnitude of dystrophy was underestimated by the reported lateral curvature as it does not reflect the vertebral dislocation between T3/T4. Dystrophic type NF-1 was confirmed in this patient due to this dislocation and multiple rib penciling and was substantiated by dural ectasia and posterior vertebral scalloping on CT and plain X-ray images.

That dystrophic type NF-1 is progressive with documented poor bone healing is non-contentious. Therefore, in treating such patients, the pertinent questions are that despite persistent progression, which surgical approach and what length of fusion will achieve stable, long-term correction with a better outcome? In this report, we used a staged combined anterior-posterior approach for the correction of angular kyphoscoliosis. To maximize the long-term stability, we determined that placing an anterior rib strut graft on the concave curvature would produce superior results compared to those with a convex-sided approach. The concave approach has an inherently higher risk of morbidity, including an increased risk of massive blood loss, compared with the convex approach. In a case reported by $\mathrm{Hsu}$, et al., an anterior approach was deemed impossible due to excessive plexiform venous channels surrounding the vertebral bodies [9]. Our patient also presented with a highly vascularized plexiform neurofibroma 
Citation: Lee J, Kawakami N, Tauchi R, et al. (2019) Surgical Treatment of Neurofibromatosis-1 with Anterior-Posterior Fusion and Concave Strut Graft for Dystrophic Kyphoscoliosis with Dislocation: A Case Report with 10-Year Follow Up. J Orthop Surg Tech 2(1):74-79
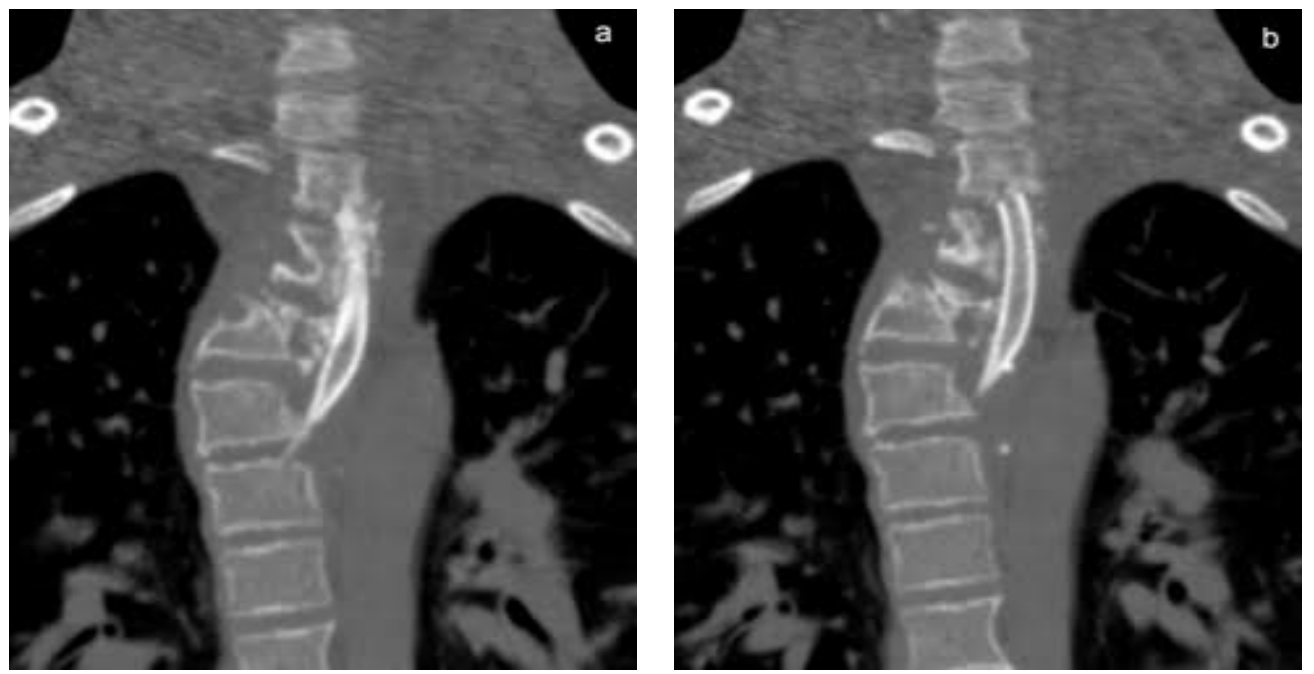

Figure 8: Postoperative CT images of T2-T6 rib strut grafting on the concave side of the thoracic curvature.

located in the anterior space requiring partial resection. Additionally, the anterior concavity in the cervical spine provided an anatomical region for displacing the great vessels, trachea, and esophagus, considerably increasing the risk of intraoperative complications. Despite the substantial risks, a concave approach was attempted to maximize regional stability due to potential for catastrophic neurological consequences with further destabilization. Scoliosis correction prior to anterior bone graft along the concave side can improve the concavity to approach more safety. A case report of kyphoscoliosis progression, despite anterior-posterior fusion for thoracolumbar subluxation, attributed the progression to lack of osseous union [9]. In our case, strut placement to correct the concavity allowed transference of force from the dislocated area (curve apex) to the more cranial and caudal vertebral bodies encouraged fusion through compressive forces (Figure 8) [10]. We speculated that this force was fundamental to maintaining spinal cord integrity and curvature stability over 10 years despite dystrophic progression. A drop in MEP at the time of corpectomy occurred when correcting the deformity, but no other significant intra- or postoperative complications including dural hematomas, neurological deficits, or nonunion occurred following any of the five surgeries.

Five surgeries were performed over the 10-year period. The third and fourth surgeries were performed to salvage the PJK of $18^{\circ}$, which developed by the fourth postoperative year. PJK is a common postoperative complication of spinal fusion with a reported incidence between $20 \%$ and $46 \%$ arising secondary to increased junctional stress concentration $[11,12]$. The proximal junction angle is the angle between the caudal endplate of the upper instrumented vertebra (UIV) to the cephalad endplate of two vertebrae proximal to the UIV $[11,13]$. PJK occurs at a point at which the junctional angle is $>10^{\circ}$ and there has been $a>10^{\circ}$ change in kyphosis from the corresponding preoperative angle [14].

The risk of PJK in our case was considered when determining the fusion length prior to the first surgery. Significant cervicothoracic dural ectasia with adjacent vertebral scalloping suggested that a proximal fusion level not incorporating the eroded region would likely lead to bony instability proximal to the UIV [8]. With the objective of minimizing operation time, blood loss, and the risk of intra- and postoperative complications, treatment priority was given to selectively stabilizing the thoracic dislocation. Furthermore, while cervical mobility was severely impacted, the course of dural expansion was not halted by preventative fusion [8]. Therefore, the posterior instrumentation was not extended to bypass the areas of cervical erosion. Subsequent dural expansion and further bony erosion led to the development of PJK, which was stabilized using a fibular graft via anterior-posterior fusion with a fibula graft. Further dystrophic progression was monitored until substantial bone thinning in the cervicothoracic zone prompted the most recent augmentation surgery.

According to the classification system developed by Yagi, et al., the ultimate mechanism by which PJK develops in cases of non-dystrophic curvature correction may be secondary to disc and ligamentous failure, bone failure, or implant/bone interface failure [11]. However, in this case, it is likely that postoperative increases in junctional stress exacerbated the progressive regional dystrophy causing PJK 4 years postoperatively.

This study was a case report, which limits our conclusions. However, this study was informative to the spine surgeons to demonstrate long-term surgical outcomes over more than 10 years in patients with severe kyphoscoliosis due to NF-1.

\section{Conflict of Interests}

Authors do not have any conflict of interests related to this work

\section{Acknowledgements}

We would like to acknowledge Sachiko Hiraiwa, Naoko Kojima, and Moeko Nishimura for their help in preparing $X$-Rays and clinical charts. The authors would also like to thank Enago (www.enago.jp) for the English language review. 
Citation: Lee J, Kawakami N, Tauchi R, et al. (2019) Surgical Treatment of Neurofibromatosis-1 with Anterior-Posterior Fusion and Concave Strut Graft for Dystrophic Kyphoscoliosis with Dislocation: A Case Report with 10-Year Follow Up. J Orthop Surg Tech 2(1):74-79

\section{References}

1. Delucia TA, Yohay K, Widmann RF (2011) Orthopaedic aspects of neurofibromatosis: Update. Current Opinion in Pediatrics 23: 46-52.

2. Tsirikos Al, Saifuddin A, Noordeen MH (2005) Spinal deformity in neurofibromatosis type-1: Diagnosis and treatment. European Spine Journal 14: 427-439.

3. Feldman DS, Jordan C, Fonseca L (2010) Orthopaedic manifestations of neurofibromatosis type 1. Journal of the American Academy of Orthopaedic Surgeons 18: 346-357.

4. Stone JW, Bridwell KH, Shackelford GD, et al. (1987) Dural ectasia associated with spontaneous dislocation of the upper part of the thoracic spine in neurofibromatosis. A case report and review of the literature. Journal of Bone \& Joint Surgery American 69: 1079-1083.

5. Winter RB (1991) Spontaneous dislocation of a vertebra in a patient who had neurofibromatosis. Report of a case with dural ectasia. Journal of Bone \& Joint Surgery American 73: 1402-1404.

6. Kim KT, Lee SH, Suk KS, et al. (2007) Spontaneous vertebral column dislocation in Neurofibromatosis - A case report. Journal of Korean Orthopaedic Association 42: 822-827.

7. Stoker GE, Lenke LG, Dorward IG (2012) Posterior vertebral column resection for the treatment of dystrophic kyphosis associated with type-1 neurofibromatosis: A case report and review of the literature. Spine 37: E1659-E1664.

8. Jain VV, Lykissas MG, Crawford AH (2015) Neurofibromatosis. In: The Growing Spine: Management of Spinal Disorders in Young Children, $\left(2^{\text {nd }} e d n\right), 265-291$.

9. Hsu LC, Lee PC, Leong JC (1984) Dystrophic spinal deformities in neurofibromatosis. Treatment by anterior and posterior fusion. Journal of Bone \& Joint Surgery British 66: 495-499.

10. Bradford DS, Winter RB, Lonstein JE, et al. (1977) Techniques of anterior spinal surgery for the management of kyphosis. Clinical Orthopaedics Related Research 128: 129-139.

11. Yagi M, Akilah KB, Boachie-Adjei O (2011) Incidence, risk factors and classification of proximal junctional kyphosis: surgical outcomes review of adult idiopathic scoliosis. Spine 36: E60-E68.

12. Cecchinato R, Berjano P, BassaniR, et al. (2015) Osteotomies in proximal junctional kyphosis in the cervicothoracic area. European Spine Journal 1: S31-S37.

13. Maruo K, Ha Y, Inoue S, et al. (2013) Predictive factors for proximal junctional kyphosis in long fusions to the sacrum in adult spinal deformity. Spine 38: E1469-E1476.

14. McClendon J Jr, O'Shaughnessy BA, Sugrue PA, et al. (2012) Techniques for operative correction of proximal junctional kyphosis of the upper thoracic spine. Spine 37: 292-303. 